\title{
Amorphous phase behavior and crystalline morphology in blends of poly(vinyl methyl ether) with isomeric polyesters: poly(hexamethylene adipate) and poly(e-caprolactone)
}

\author{
Eamor M Woo, Ying-Hsuan Chou, Wan-Jing Chiang, I-Tzu Chen, I-Han Huang and Nai-Tzu Kuo
}

Two isomeric polyesters, poly(1,6-hexamethylene adipate) (PHA) and poly( $\varepsilon$-caprolactone) (PCL), were each blended with amorphous poly(vinyl methyl ether) (PVME) and investigated and compared in the crystalline and amorphous phases. The amorphous phase, $T_{\mathrm{g}}$, and crystalline morphology of the PVME/PHA blend were compared with those of classical PVME/PCL. Both blend systems exhibit a similar peculiar asymmetry with positive deviation in $T_{\mathrm{g}}$-composition relationships, and both are similarly miscible, with the PVME/PHA blend exhibiting $\chi_{12}=-0.21$; for PVME/PCL, $\chi_{12}=-0.20$. These values indicate that both blends are similarly miscible but have only quite weak interactions in the amorphous phase. Neat PHA shows a regime-I to -II transition, but neat PCL exhibits a regime-II to -III transition. On blending with PVME (at 20 wt\%), both PVME/PCL and PVME/PHA blends undergo changes in crystallization temperature $\left(T_{c}\right)$. Dendritic spherulites in the high- $T_{c}\left(46^{\circ} \mathrm{C}\right.$ or above)crystallized PVME/PHA blend assume a straight-stalk pattern (chrysanthemum flower pestles or banana stems). In contrast to the similarities in amorphous-phase miscibility, the crystalline phases of these two blends differ significantly. When crystallized at high $T_{\mathrm{c}}\left(46^{\circ} \mathrm{C}\right.$ or above), the PVME/PCL (20/80) blend assumes a fluffy feather-like dendritic pattern, which is dramatically different from the straight-stalk dendrites in the PVME/PHA (20/80) blend.

Polymer Journal (2010) 42, 391-400; doi:10.1038/pj.2010.18; published online 24 March 2010

Keywords: blend miscibility; crystalline morphology; dendrites; PCL; PHA; PVME

\section{INTRODUCTION}

Poly( $\varepsilon$-caprolactone) (PCL) is known to be miscible with many other polymers through hydrogen bonding, leading to miscible blends with specific interactions. However, the phase behavior in blends of PCL with nonhydrogen polymers, such as ether- or carbonyl-containing polymers, is not always easy to interpret. Blends of poly(vinyl methyl ether) (PVME) with polyesters have also been attempted by many investigators, but they are not widely reported, probably owing to the peculiar phase behaviors often encountered in these blends. The moststudied system involving PVME and polyesters is the PVME/PCL blend, ${ }^{1-4}$ but its phase behavior and miscibility are still quite undefined. In addition, there have been no reports on blends of PVME with polyesters other than PCL until a recent work from our laboratory, ${ }^{5}$ which demonstrated that PVME can be miscible with polyesters the structures (main backbone) of which are within a certain range. However, the phase behavior has not yet been clearly revealed in the reported PVME/PCL blend. For example, the interaction strength for the PVME/PCL blend system has not been characterized; the blend $T_{\mathrm{g}}$ (especially for PCL-rich compositions) shows a peculiar positive deviation from linearity and several other unsettled issues in its phase behavior. Even more peculiarly, melting point elevation, rather than depression, in the PVME/PCL blend has been reported. ${ }^{1}$ Such an interesting yet peculiar deviation from normal in the phase behavior of the PCL/PVME blend has not been observed in other blend systems involving PCL, such as PCL/phenoxy, ${ }^{6} \mathrm{PCL} /$ poly $(p \text {-vinyl phenol })^{7}$ or $\mathrm{PCL} / \operatorname{tannin},{ }^{8}$ all of which show specific interactions between the constituent polymers. Conventional notions are that strong specific interactions (H-bondings or ionic interactions and so on) are more likely to lead to positive deviations in $T_{\mathrm{g}}$-composition relationships for blends the miscibility of which is based on such strong interactions. The positive interactions in the $T_{\mathrm{g}}$-composition relationships for weakly interacting PCL/PVME blends must originate from factors other than interaction strength.

PVME is a water-soluble polymer, which indicates that it is capable of $\mathrm{H}$-bonding with water molecules and, therefore, probably with other hydroxyl-containing molecules or polymers. Thus, intuitively, miscibility in blends of PVME with hydroxyl- or phenol-containing polymers can be expected. Phase behavior with miscibility through apparently specific interactions is common when PVME is blended with H-bonding-type polymers. For example, PVME, which contains 
a pendant ether (-O-) group, is commonly known to be miscible with several polymers containing hydroxyl $(-\mathrm{OH})$ groups, providing suitable sites for specific interactions. In addition, PVME is known to be miscible with the hydroxyl ether of bisphenol-A (that is, phenoxy), showing a sharp and composition-dependant $T_{\mathrm{g}} \cdot{ }^{9}$ PVME is also known to be miscible with hydroxyl-containing polymers such as poly( $p$-vinyl phenol). ${ }^{10}$ Many other examples can be cited. However, by contrast, PVME exhibits interestingly peculiar phase behaviors when it is blended with polymers of lesser polarity. PVME has been demonstrated to be miscible with some acrylic polymers. The blends of PVME with carboxyl-containing polymers have attracted much interest. As an extension of this interest, our previous studies ${ }^{11}$ found miscibility in poly(benzyl methacrylate) (PBzMA)/PVME and poly (phenyl methacrylate)/PVME blends. However, the phase behaviors of the above blends, which have lesser interactions, are typified by two features: (a) a relatively low lower-critical-solution-temperature (LCST), and (b) a phase of cast samples strongly affected by solvents and casting temperatures, owing to the kinetic processes. For example, poly(ethyl acrylate) or poly(butyl acrylate) is miscible with PVME. ${ }^{12}$

A previous study, ${ }^{5}$ as well as other studies in literature, pointed out that crystallinity in the polyester constituents of blends may cause significant asymmetry and peculiarity (cusp, or occasionally positive deviation) in the $T_{\mathrm{g}}$-composition relationships for the claimed miscibility. Such behavior must be dealt with carefully in the interpretation of phase behavior and interaction strength. This study, in continuation of previous work, ${ }^{5}$ was intended to further probe the phase behavior of a blend of PVME with PCL in comparison with that of PCL with poly(hexamethylene adipate) (PHA), which is a polyester having an isomeric structure with PCL. Although the PVME/PCL blend has been studied previously and results have been reported in literature, the abrupt and positive deviation from linearity in the $T_{\mathrm{g}}$, especially for PCL-rich compositions, was never adequately interpreted. Residual crystallinity in the PCL/PVME blend has been cited as a factor leading to peculiar phase behavior. As a polyester that is structurally similar to PCL, PHA was used as a comparison because PHA has a stronger tendency for crystallization than PCL. Both PCL and PHA are capable of forming ring bands in a narrow temperature range, but the crystalline morphologies at high crystallization temperature $\left(T_{\mathrm{c}}\right)$ differ in these two polyesters. The regime behavior and crystalline morphologies for the PVME/PCL blend were compared with those for the PVME/PHA blend. In addition, transitions and accompanying changes in crystal structures from spherulites to different shapes of crystal aggregates are widely reported in classical literature; however, few of these address the changes in ring band structures in the corresponding transition of kinetic regimes. This study provides timely probes to fill the inquiry gap.

\section{EXPERIMENTAL PROCEDURE}

\section{Materials and preparation}

Blend systems in this study were designed in such a pattern that they comprised two polymers, one having a pendant oxy ether group (vinyl ether) and the other having a carbonyl group on the backbone (polyester). PVME (Scientific Polymer Products, New York, NY, USA), a water-soluble polymer, was received as a $50 \mathrm{wt} \%$ solution in water. The average molecular weight (by gel permeation chromatography) of PVME is $M_{\mathrm{w}}=64000$, and $M_{\mathrm{n}}=32000 \mathrm{~g} \mathrm{~mol}^{-1}$ (PI=1.99) with $T_{\mathrm{g}}=-22.5^{\circ} \mathrm{C}$. PVME was purified three times by re-dissolution in water and precipitation and was dried under vacuum at $110^{\circ} \mathrm{C}$ for $72 \mathrm{~h}$. The two polyesters, poly(hexamethylene adipate) (PHA) and PCL, which have isomeric structures relative to each other and backbone average $\mathrm{CH}_{2} / \mathrm{CO}$ ratios equal to 5, were used for blending with PVME. PHA, with $T_{\mathrm{g}}=-65^{\circ} \mathrm{C}, T_{\mathrm{m}}=55.3^{\circ} \mathrm{C}$ and $M_{\mathrm{w}}=15000 \mathrm{~g} \mathrm{~mol}^{-1}$, was obtained from Aldrich (Milwaukee, WI, USA). PCL, with a structure isomeric to PHA, was purchased from Aldrich, with an approximate $M_{\mathrm{w}}=20000 \mathrm{~g} \mathrm{~mol}^{-1}$ and literature values of $T_{\mathrm{g}}=-70{ }^{\circ} \mathrm{C}$ and $T_{\mathrm{m}}=60^{\circ} \mathrm{C}$. It should be noted that, in addition to the similarities in the backbone average $\mathrm{CH}_{2} / \mathrm{CO}$ ratios, the physical constants $\left(T_{\mathrm{g}}, T_{\mathrm{m}}\right.$ and $\left.M_{\mathrm{w}}\right)$ for isomeric PCL and PHA are also very similar. However, the chemical structure of PHA allows it to have a crystallization tendency much greater than that of PCL.

Before use, PHA was fractionated and purified by dissolving in THF ( $4 \mathrm{wt} \%$ ), then precipitated in iced methanol, filtered and dried. The molecular weight of purified PHA was then measured using gel permeation chromatography (Waters, Milford, MA, USA), and $M_{\mathrm{w}}=15000 \mathrm{~g} \mathrm{~mol}^{-1}$ was obtained. The molecular weights of the PCL and PHA materials used in this study were chosen so that they would have similar $M_{\mathrm{w}}$ for the comparison of the phase behaviors of their respective blends. Blend samples were prepared using solvent mixing, followed by film casting. The solvent tetrahydrofuran was used in its as-received state (high-performance liquid chromatography grade). Solvent in the polymer mixtures during film casting was first allowed to evaporate by slow convection; thereafter, the dried films were further fully de-gassed under vacuum at $70-80^{\circ} \mathrm{C}$ for $72 \mathrm{~h}$ to drive off the residual solvent.

Sample films were generally prepared as follows: a solution of blend was prepared by dissolving it in solvent $(2-4 \mathrm{wt} \%)$. This solution was then cast on glass slides for polarized optical microscope (POM) and scanning electron microscope observations. After solution casting and drying, the cast films on glass slides were briefly melted to liquid at $\sim 100^{\circ} \mathrm{C}$ and then rapidly brought to an isothermal $T_{\mathrm{c}}$ for full crystallization or dynamic growth observation. The film thickness, after subtracting for the glass slide, was estimated to be $\sim 5-10 \mu \mathrm{m}$. To enhance the contrast, a set of samples were washed with pure water to etch out the water-soluble amorphous PVME component in the blends in order to expose the crystalline polyester's lamellar textures.

\section{Apparatus and procedures}

Differential scanning calorimetry. Thermal transitions of blends were characterized with a differential scanning calorimeter (DSC-7, Perkin-Elmer, Waltham, MA, USA) equipped with an intracooler for quenching and cooling. Before each $T_{\mathrm{g}}$ measurement, samples were first homogenized in DSC cells (sample holders) by heating to about $80^{\circ} \mathrm{C}$ and then quenched to subambient temperatures $\left(-70^{\circ} \mathrm{C}\right)$ before initiating the second scans at $20^{\circ} \mathrm{C} \mathrm{min}^{-1}$. Before the DSC runs, the temperature and heat of transition of the instrument were calibrated with indium and zinc standards. A continuous nitrogen flow in the DSC sample cell was maintained. Typically, a heating rate of $10^{\circ} \mathrm{Cmin}^{-1}$ was used to measure $T_{\mathrm{m}}$, whereas a heating rate of $20^{\circ} \mathrm{C} \mathrm{min}^{-1}$ was used for $T_{\mathrm{g}}$ characterization. A high heating rate of $40^{\circ} \mathrm{C} \mathrm{min}^{-1}$ was used in blend samples with high crystallinity to enhance the $T_{\mathrm{g}}$ signals for the amorphous phase. For comparison, wherever $40^{\circ} \mathrm{C} \mathrm{min}^{-1}$ was used for the blends, the same heating rate was also used to acquire $T_{\mathrm{g}}$ data in the corresponding neat polymer samples.

Optical microscopy. A polarized light microscope (Nikon Optiphot-2, POL, Tokyo, Japan) with a Nikon DS-5M-U CCD digital camera was used to observe the phase structure of as-cast blends and to monitor the phase transition of blends on heating. Blend samples were cast as thin films (solvent casting at controlled temperatures and vacuum drying) and placed on a microscope heating stage (Linkam THMS-600 with TP-92 temperature programmer, Linkam Scientific Instruments Ltd, Tadworth, UK) for optical microscopic examination.

Growth rate measurements. The radial growth of spherulites was recorded, and growth rates were measured using a polarized optical microscope (POM, Nikon Optiphot-2) equipped with a temperature-controlled hot stage, a Nikon chargecoupled device CCD digital camera and automatic image processing software. The magnification and scales were automatically calibrated by the software. Spherulite growth kinetics was based on samples of thin films $(\leqslant 5 \mu \mathrm{m})$ on glass substrates. The growth rates below $40{ }^{\circ} \mathrm{C}$ were found to be too fast to be accurately recorded. The spherulite growth rates of PCL and PVME/PCL were determined by measuring the spherulitic radii, $R$, as a function of time during isothermal crystallization in the hot stage of a polarizing microscope.

\section{RESULTS AND DISCUSSION}

\section{Amorphous phase behavior of PVME/PCL vs PVME/PHA}

Preliminary screening by optical microscopy techniques revealed that both blends of PVME/PCL and PVME/PHA were clearly above their 
respective crystalline polyester's $T_{\mathrm{m}}$. They remained in this condition until reaching the temperature of degradation $\left(250{ }^{\circ} \mathrm{C}\right.$ or above), and they showed no sign of LCST phase transition. These two blend systems were further analyzed and compared in terms of thermal behavior. Thermal analysis using DSC was performed on these two blend systems for further $T_{\mathrm{g}}$ evidence on phase homogeneity. The thermal transitions, enthalpy of fusions, and $T_{\mathrm{g}}$-composition relationships for these two blend systems, PVME/PHA and PVME/PCL, at various compositions are compared. Figure 1 shows DSC thermograms for the PVME/PCL blends of 11 compositions, as indicated in the curves. Note that a $40{ }^{\circ} \mathrm{C} \mathrm{min}{ }^{-1}$ DSC scan rate was used to obtain good $T_{\mathrm{g}}$ signals, a strategy that was regarded as necessary for dealing with the hard-to-suppress crystallinity in low $T_{\mathrm{g}} \mathrm{PCL}$ or its blends. The DSC result provides clear evidence of a single $T_{\mathrm{g}}$ for the PVME/ PCL blends of various compositions, as indicated in the curves. The $T_{\mathrm{g}}$

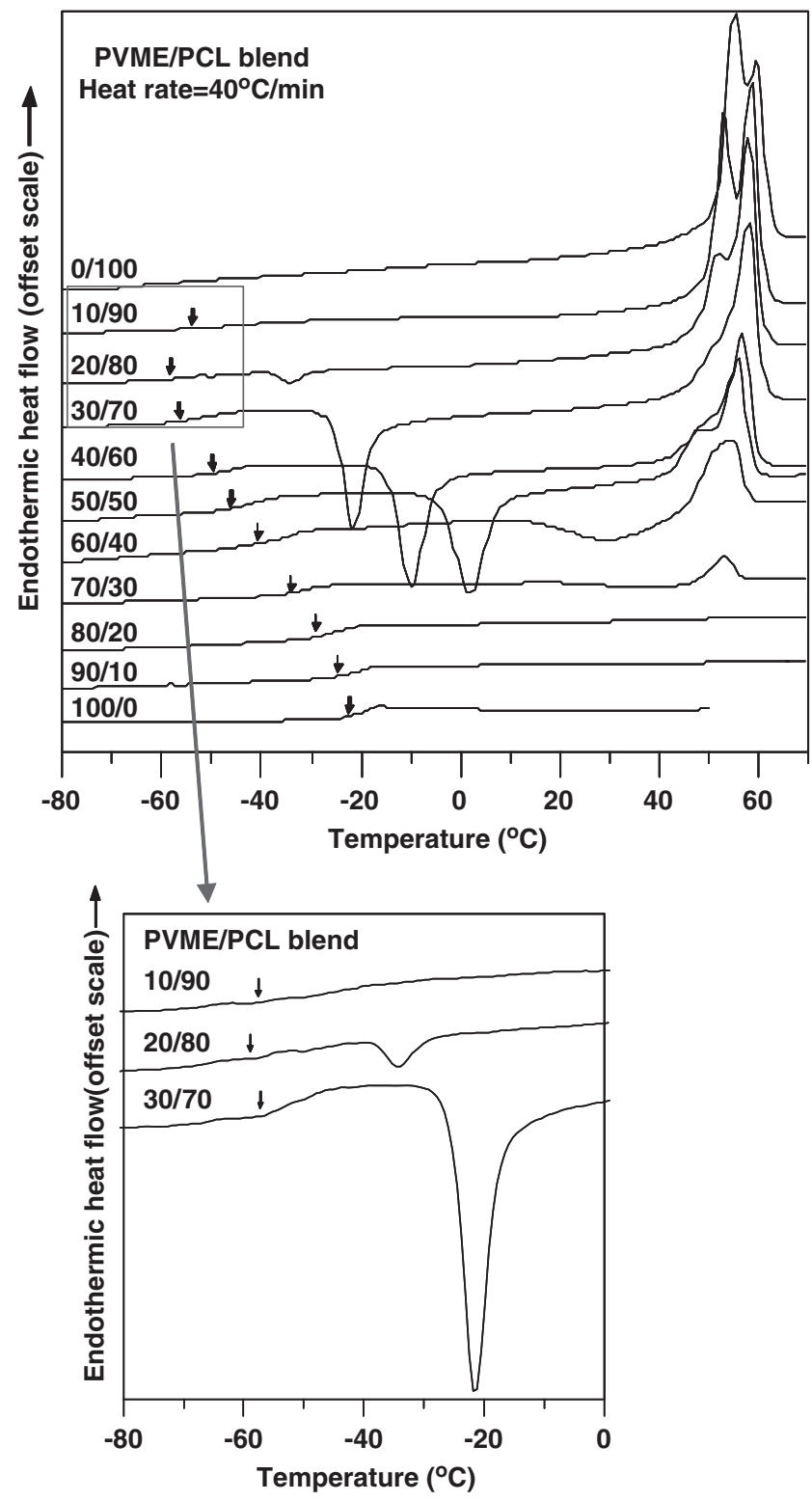

Figure 1 DSC thermograms for the PVME/PCL blends of 11 compositions. Inset: enlarged portions of compositions (10/90, 20/80, 30/70) with less-distinct $T_{\mathrm{g}}$ signals. A full color version of this figure is available at the Polymer Journal online. signals for the blends of PCL-rich compositions, owing to the hardto-depress crystallinity in the samples, are less clear than those for the other compositions; however, a general trend is quite apparent in which only a single $T_{\mathrm{g}}$ is present in each of the compositions and $T_{\mathrm{g}}$ increases with the PVME content in the blends. For better visualization, the inset graph on the bottom shows the enlarged portions of the DSC traces for the blends $(10 / 90,20 / 80,30 / 70)$ with less-distinct $T_{\mathrm{g}}$ signals.

For comparison, a similar thermal analysis characterization was performed to reveal a single $T_{\mathrm{g}}$ and the composition dependence of the $T_{\mathrm{g}}$ values of blends for another blend system, PVME/PHA. Figure 2 shows DSC thermograms for the PVME/PHA blends of 11 compositions. Note again that similar to the PVME/PCL blend system discussed in the previous figure, the $40{ }^{\circ} \mathrm{C} \mathrm{min}^{-1}$ DSC scan rate was used to obtain better $T_{\mathrm{g}}$ signals. A higher scanning rate in this case was

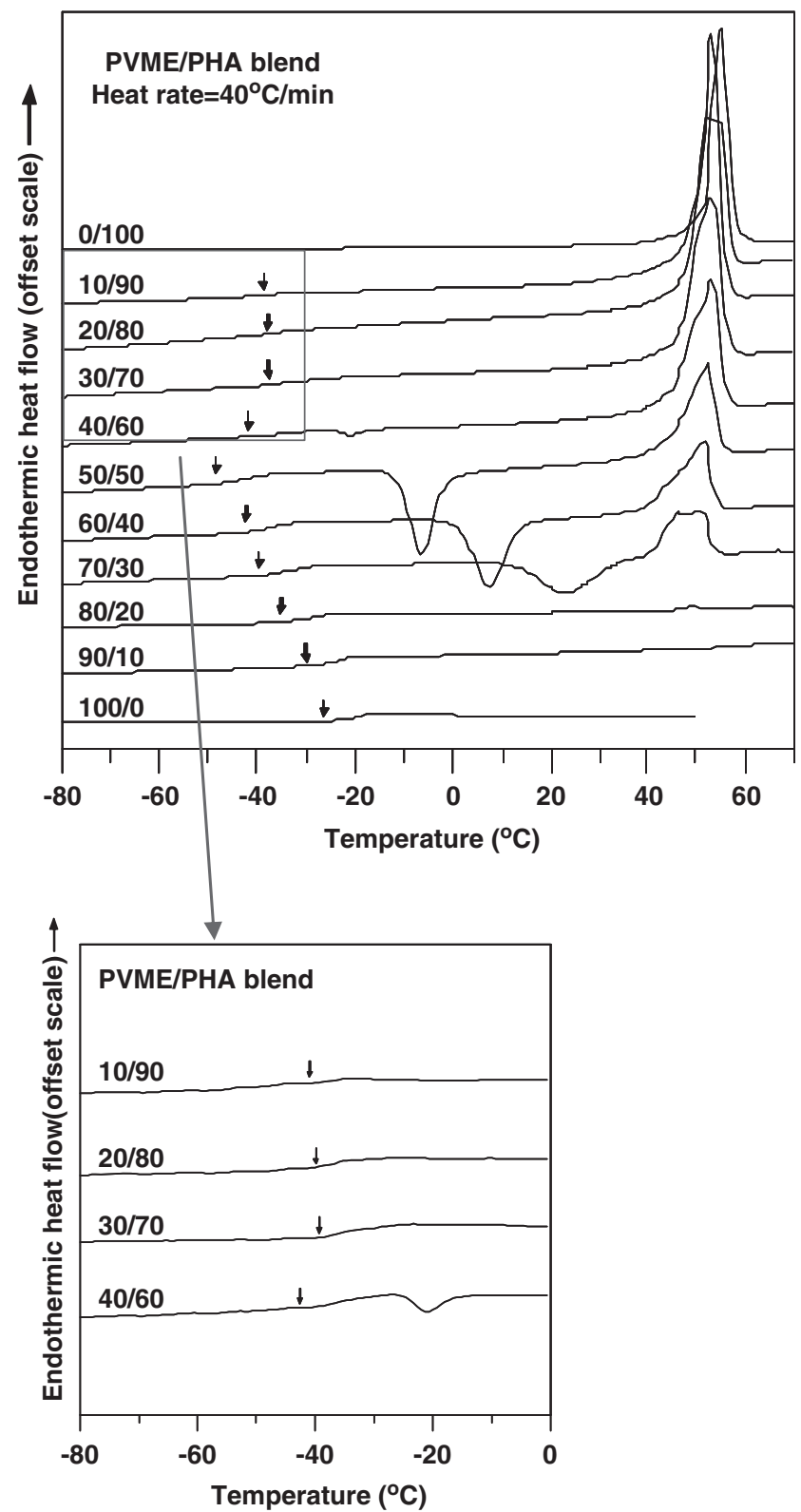

Figure 2 DSC thermograms for PVME/PHA blends of 11 compositions. Inset: enlarged portions of compositions (10/90, 20/80, 30/70) with less-distinct $T_{\mathrm{g}}$ signals. A full color version of this figure is available at the Polymer Journal online. 


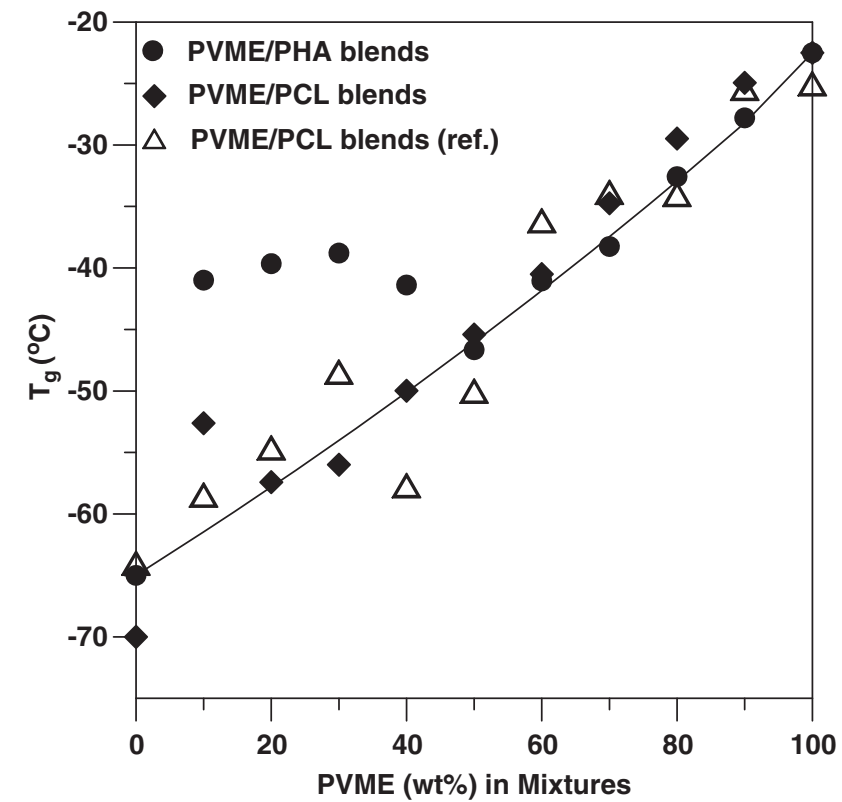

Figure 3 Comparison of $T_{\mathrm{g}}$ values vs composition for PVME/PCL and PVME/ PHA blends. Reference data (open triangles) were taken from the cited references for comparison.

regarded as necessary for dealing with the hard-to-suppress crystallinity in PHA or its blends with low $T_{\mathrm{g}}$ values. The DSC result for the PVME/PHA blend again reveals clear evidence of a single and composition-dependent $T_{\mathrm{g}}$ for various compositions. The inset shows enlarged portions of the DSC traces for the blends with lessdistinct $T_{\mathrm{g}}$ signals. Note that the phase behavior for these two blend systems is apparently quite similar.

The $T_{\mathrm{g}}$-composition relationship usually provides insight into correlations with molecular or segmental interactions or scales of phase homogeneity in blends, both of which will be shown and compared with the literature results in a later plot. Figure 3 shows comparative plots of $T_{\mathrm{g}}$ values vs composition for PVME/PCL and PVME/PHA blends; the literature $T_{\mathrm{g}}$ values for the PVME/PCL blend system were plotted in the same figure for comparison (open triangle data from cited references) with the data obtained in this study for the same PVME/PCL blend. A plot of the $T_{\mathrm{g}}$ values of the blend as a function of composition for the PVME/PCL blend system was constructed. There are two peculiar points in phase behavior, as revealed by the $T_{\mathrm{g}}$ vs composition plots. First of all, the $T_{\mathrm{g}}$ values of blends are generally located at or near the curve predicted by the linearity rule $\left(T_{\mathrm{g}}=\mathrm{w}_{1} T_{\mathrm{g} 1}+\mathrm{w}_{2} T_{\mathrm{g} 2}\right)$ or the Fox model $\left(T_{\mathrm{g}}=\mathrm{w}_{1} / T_{\mathrm{g} 1}+\mathrm{w}_{2} / T_{\mathrm{g} 2}\right)$. Second, positive deviation from linearity is noted, especially for PCL-rich blend compositions (PVME at 10-20 wt\% in blends). This is especially apparent in PVME/PCL blends with PVME contents equal to or lower than $20 \mathrm{wt} \%$. A plot of the $T_{\mathrm{g}}$ values of the blends as a function of composition for the PVME/PHA blend system shows an abrupt discontinuity at high PHA contents (PVME wt $\%$ equal to or $<40 \mathrm{wt} \%$ ). On usage of the same quenching schemes, the PHA in the PVME/PHA blend tends to be more crystallizable than the PCL in the PVME/PCL blend, as discussed earlier. Thus, the abrupt positive deviation of $T_{\mathrm{g}}$ from linearity for the PHA-rich blend compositions is more pronounced, and the positive deviation spreads in a wider range of PHA-rich compositions (90-60 wt\% PHA in blends). By comparison, for the PVME/PCL blend, the range of positive deviation is narrower and is restricted to between 90 and $80 \mathrm{wt} \%$ PCL in the blends. Apparently, the higher

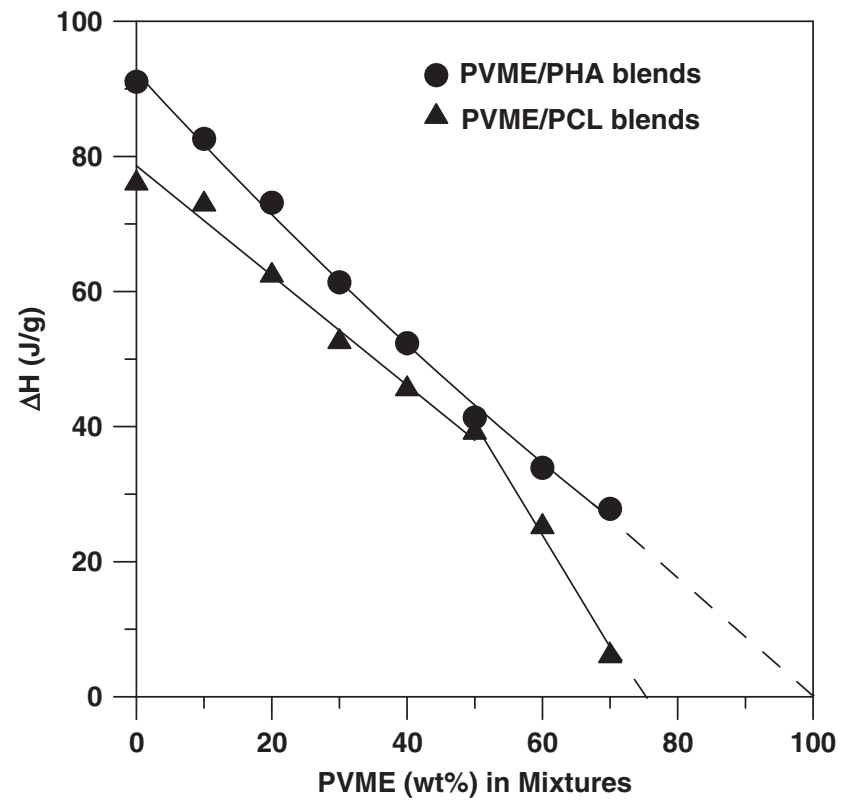

Figure 4 Heats of fusion $\left(\Delta H_{f}\right)$ as functions of PVME wt\% in PVME/PCL and PVME/PHA blends.

crystallinity and stronger tendency for crystallization of PHA in comparison with PCL accounts for the difference in $T_{\mathrm{g}}$ behavior. The reason the positive deviation of $T_{\mathrm{g}}$ from the linear dependence on composition is claimed to be 'peculiar' is that not all blends having a crystalline phase would behave this way. Blends of PCL with strongly interacting amorphous polymers such as poly(vinyl p-phenol) or phenoxy, for example, do not show a positive deviation of $T_{\mathrm{g}} \mathrm{vs}$ composition (in the PCL-rich range). The differences are due to the interaction strength between PCL (or PHA) and amorphous polymers. Greater interactions tend to more effectively suppress the crystallinity of PCL (or PHA) in blends, and positive deviations of the $T_{\mathrm{g}}$-composition relationship above linearity are less likely to occur in blends of PCL (or PHA) with amorphous polymers. Thus, generally, the $T_{\mathrm{g}}$-composition relationships for hydrogen-bonding systems, such as miscible poly( $p$-vinyl phenol)/PCL or phenoxy/PCL blends, display typical negative deviation from the Fox-model relationship, with no abrupt positive $T_{\mathrm{g}}$ deviation from linearity in any of the blend composition ranges. ${ }^{6,7}$

Figure 4 shows the heat of fusion $\left(\Delta H_{\mathrm{f}}\right)$ as a function of PVME wt $\%$ in PVME/PCL and PVME/PHA blends. Both blends show decreasing $\Delta H_{\mathrm{f}}$ on increasing the weight fraction of PVME. The dependence of the enthalpy of transitions on blend composition yields clues of the strength of intermolecular interactions. For blends of noninteracting or weakly interacting components in which one is crystallizable and the other is not, changes of the blend's heat of melting with respect to composition are expected to be linearly dependent on the weight fraction of the crystallizing species. Usually, a blend's heat of fusion showing linear dependence on the weight fraction of the crystallizing species suggests either a phase of two weakly interacting species or two separate phases with no interactions. The $\Delta H_{\mathrm{f}}$ values of blends show linear additive dependence on the weight fraction of PHA content in the blends, indicating that the segmental interactions between these two constituent polymers are not strong. For the PVME/PCL blend, a slight nonlinearity is noted. By comparison, the depression of PCL crystallinity is more significant in the PVME/PCL blend than in the PVME/PHA blend at the same PVME loadings. 
An estimation of the interaction parameters for PVME/PCL and PVME/PHA blends was attempted by following the classical standard procedures. The equilibrium $T_{\mathrm{m}}^{\mathrm{o}}$ shows a continuous decreasing trend with corresponding increases in the amorphous polymer in the blends. The quantitative comparison of the interaction strength for these blends is discussed in the following section. The equilibrium melting temperatures for neat polyesters (PCL and PHA) and their blends of various compositions were then used for the estimation of the interaction strength between the polyester and PVME as a miscible diluent, which is based on the relationship according to the FloryHuggins treatment: ${ }^{13,14}$

$\left(\frac{1}{\left.T_{\mathrm{m}}^{\mathrm{o}} \text { (blend }\right)}-\frac{1}{T_{\mathrm{m}}^{\mathrm{o}}(\text { pure })}\right)=-\frac{R V_{2}}{\Delta H^{\mathrm{o}} V_{1}}\left[\frac{\ln \phi_{2}}{m_{2}}+\left(\frac{1}{m_{2}}-\frac{1}{m_{1}}\right) \phi_{1}+\chi_{12} \phi_{1}^{2}\right]$

Equation (1) can be further re-arranged to:

$$
\begin{aligned}
& -\left[\frac{\Delta H^{\mathrm{o}} V_{1}}{R V_{2}}\left(\frac{1}{T_{\mathrm{m}}^{\mathrm{o}}(\text { blend })}-\frac{1}{T_{\mathrm{m}}^{\mathrm{o}}(\text { pure })}\right)+\frac{\ln \phi_{2}}{m_{2}}+\left(\frac{1}{m_{2}}-\frac{1}{m_{1}}\right) \phi_{1}\right] \\
& =\chi_{12} \phi_{1}^{2}
\end{aligned}
$$

where $T_{\mathrm{m}}^{\mathrm{o}}$ (blend) and $T_{\mathrm{m}}^{\mathrm{o}}$ (pure) are the equilibrium melting points of the blend and pure crystallizable polymers, respectively, $\Delta H^{\circ}$ is the heat of fusion of the fully crystalline polymers, $V$ is the molar volume of the repeating units of polymers, and $m$ and $\phi$ are the degree of polymerization and the volume fractions of the component in the blends, respectively. Subscripts 1 and 2 refer to the amorphous and crystalline polymer, respectively, $R$ is the universal gas constant and $\chi_{12}$ is the Flory-Huggins interaction parameter. The physical constants for PVME and PCL are as follows: $\Delta H^{\mathrm{O}}=23800 \mathrm{~J} \mathrm{~mol}^{-1}$, $V_{1}=55.8 \mathrm{~cm}^{3} \mathrm{~mol}^{-1}, V_{2}=105 \mathrm{~cm}^{3} \mathrm{~mol}^{-1},{ }^{15,16} m_{1}=1103$ and $m_{2}=175$. Subscript 1 indicates PVME as the amorphous diluent, and subscript 2 indicates the crystal-melting PCL. The densities for these two constituents are $\rho_{\text {PVME }}=1.05 \mathrm{~g} \mathrm{~cm}^{-3}$ for PVME and $\rho_{\mathrm{PCL}}=1.143 \mathrm{~g} \mathrm{~cm}^{-3}$ for PCL. For PHA, the constants are $\Delta H^{\mathrm{o}}=38100 \mathrm{~J} \mathrm{~mol}^{-1}$, $V_{1}=55.8 \mathrm{~cm}^{3} \mathrm{~mol}^{-1}, \quad V_{2}=203 \mathrm{~cm}^{3} \mathrm{~mol}^{-1}, m_{1}=1103, m_{2}=65.8$ and $\rho_{\mathrm{PHA}}=1.221 \mathrm{~g} \mathrm{~cm}^{-3}$. All lines are linear and more or less go through the origin, indicating little effect from an entropic contribution in estimating the parameters.

From the analyses of the Flory-Huggins treatment and assuming that the entropy effect was negligible by dropping the second and third terms inside the bracket of Equation (2), plots of $\left(\left(1 / T_{\mathrm{mb}}\right)-\left(1 / T_{\mathrm{m}}^{\mathrm{o}}\right)\right)$ vs $\phi_{1}{ }^{2}$ and values of $\chi_{12}$ could be obtained for PVME/PHA in comparison with the PVME/PCL blend. Negative values could confirm the miscibility and favorable interactions between constituents in the blend. Similar procedures were performed on those blends that were deemed miscible according to $T_{\mathrm{g}}$-composition behavior. Figure 5 shows plots for determination of the interaction parameters for the PVME/PCL and PVME/PHA blend systems. All lines are linear and more or less go through the origin, positively indicating the validity of the assumption of little effect from an entropic contribution to the interaction parameters. The inset diagrams in Figure 5 are summary plots of $T_{\mathrm{m}}$ vs $T_{\mathrm{c}}$ for the two blend systems (PVME/PCL and PVME/ PHA) and respective extrapolations according to the Hoffman-Weeks procedures. As the procedures are standard, for brevity, the data are not repetitively shown here for the respective DSC thermograms of the crystallized blends showing a $T_{\mathrm{m}}$ for each sample crystallized at $T_{\mathrm{c}}$. Extrapolations to the $T_{\mathrm{m}}=T_{\mathrm{c}}$ line determine the equilibrium melting temperatures for neat polyesters and their blends of several different compositions (between 10-40 wt \% PVME in blends, respectively).

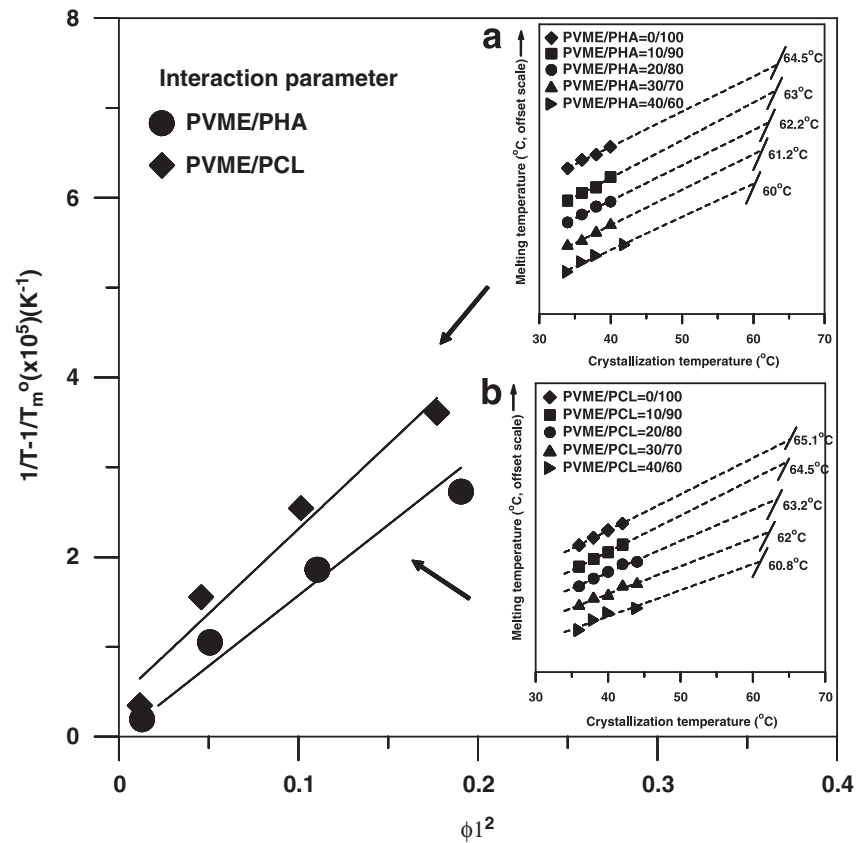

Figure 5 Flory-Huggins plots for comparing the interaction parameters of blends of PVME/PCL and PVME/PHA, respectively. Insets: diagrams illustrating the plots of $T_{\mathrm{m}}$ Vs $T_{\mathrm{c}}$ for the blend systems of (a) PVME/PCL and (b) PVME/PHA, according to the Hoffman-Weeks procedures.

From analysis of the Flory-Huggins treatment ${ }^{13,14}$ and the best-fit plot of $\left(\left(1 / T_{\mathrm{mb}}\right)-\left(1 / T_{\mathrm{m}}^{\mathrm{o}}\right)\right)$ vs $\phi_{1}{ }^{2}$, a negative value of $\chi_{12}$ was obtained as -0.20 for the PVME/PCL blend, which confirms the miscibility and favorable (though not particularly strong) interactions between the constituents. By comparing these two blend systems, a slightly more negative $\chi_{12}$ was obtained as -0.21 for the PVME/PHA blend using similar procedures as those stated earlier. The values of the interaction parameters for PVME/PCL and PVME/PHA blends are, within experimental errors, quite similar, although the parameter for the PVME/PHA blend is slightly lower than for PVME/PCL. The higher crystallinity of PHA in blends may be partly responsible for the difference. Although the positive deviation of $T_{\mathrm{g}}$ from linearity is noted as being due to the residual crystallinity in PCL, the melting points are also depressed in the PVME/PCL blend, as expected in most miscible blend systems.

Note that the values of $\chi_{12}$ for PVME/PCL that had been reported earlier in literature were somewhat conflicting, as earlier investigators used different approaches that might involve different degrees of uncertainty. ${ }^{1-4}$ Yam et al. ${ }^{1}$ used the LCST phase separation temperature to extrapolate an interaction parameter value of a very small negative at -0.00008 by assuming a reference $T_{\mathrm{A}}=7200 \mathrm{~K}$. On the other hand, ten Brinke and coworkers ${ }^{3}$ claimed that melting point elevation, rather than depression, occurred in the PCL/PVME blend. To clarify the conflicting issues, this study performed a further careful and detailed thermal analysis and observed that melting point depression, and not elevation, actually occurred in PVME/PCL and PVME/PHA blends. The values of the interaction parameters for both PVME/PCL and PVME/PHA blends were more precisely identified using the melting-point-depression method than using LCST phase separation temperatures, as the LCST was more prone to changes with respect to the $M_{\mathrm{w}}$ of polymer components in the blends. That is, even though the $T_{\mathrm{g}}$ behavior of the amorphous phase in the PVME/PCL blend shows a positive deviation from the general trend of the $T_{\mathrm{g}}$-composition relationship, its crystalline phase nevertheless shows 


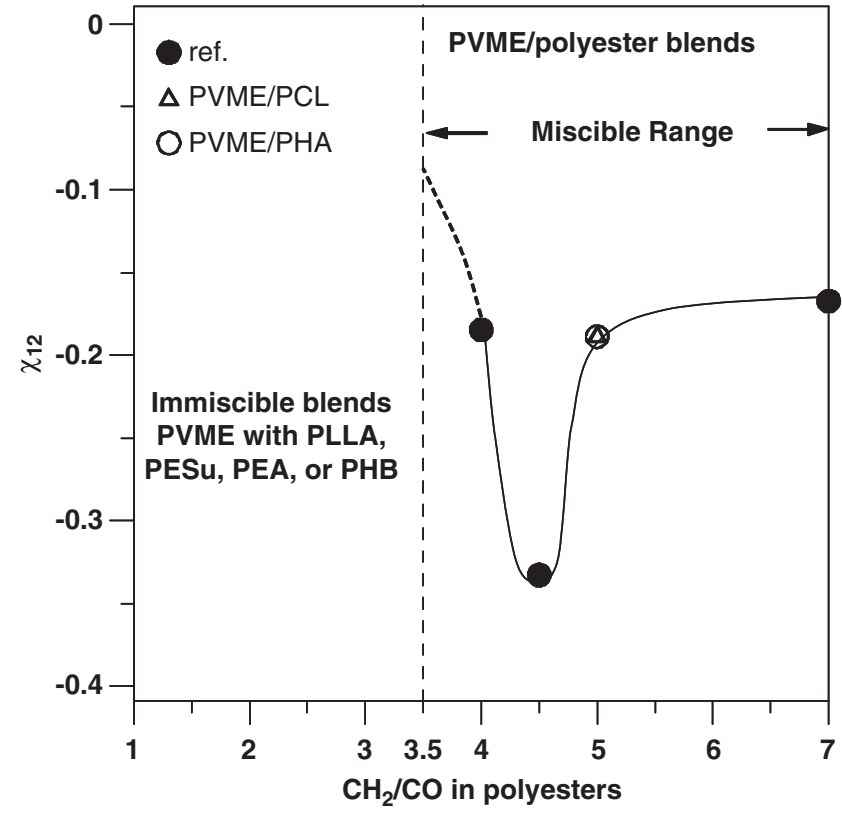

Figure 6 Interaction parameters for PVME/PCL and PVME/PHA blends, compared with blends of PVME with other polyesters of different structures. Reference data (filled circles) were taken from the cited references for comparison.

melting-point depression and a small but negative interaction parameter. The values of $\chi$ are quite comparable because PCL and PHA possess similar structures, although PHA has a higher crystallizing tendency than PCL.

An earlier study before this work ${ }^{5}$ showed that PVME can be miscible with several polyesters, and the resultant blend systems show different interaction strengths within a window of polyester structures. The polyesters with $\mathrm{CH}_{2} / \mathrm{CO}=3.5,4.0,4.5$ and 7.0 (that is, poly (trimethylene adipate), poly(butylene adipate) and poly(hexamethylene sebacate) and so on) have been found to be miscible with PVME, with the interaction strength varying with the backbone-chain structures of polyesters. PVME is known to be immiscible (phase separated) with some aliphatic polyesters or biodegradable polyesters, the chemical structures of which have low $\mathrm{CH}_{2} / \mathrm{CO}$ ratios, such as poly(ethylene succinate), poly (L-lactide), poly( $\beta$-hydroxy butyrate) or poly(ethylene adipate). A plot of interaction strength vs polyester structure shows that a maximum (that is, the greatest negative value) interaction strength is located at the blend of PVME with poly(ethylene azealate) (PEAz), a polyester with $\mathrm{CH}_{2} / \mathrm{CO}=4.5 .{ }^{5}$ However, without data of the phase behavior and interaction strength of the PVME/PCL and PVME/PHA blends (the polyester constituents of which have a $\mathrm{CH}_{2} / \mathrm{CO}$ ratio=5.0), the description of the trend of phase behavior for PVME/polyesters has remained incomplete. Blend systems of PVME/PCL and PVME/PHA furnished the required data for a more complete description. For this purpose, Figure 6 shows the phase behavior and interaction parameters of PVME/PCL and PVME/ PHA blends in comparison with other blends of PVME with polyesters of different structures. Data of interaction parameters for blends of PVME with polyesters other than PCL or PHA were taken from the cited references. ${ }^{5}$ This study provides additional data of both PVME/ PHA and PVME/PCL blends, completing a summary plot to better view the trend of variation of interaction strength with respect to the polyesters' structures. Note that the overall trend as shown in this figure is slightly different from that shown in a previous study in the literature, ${ }^{5}$ as the additional data fill in the gap for a more complete trend. Although all data in reality are discrete points for each of the polyester structures, a dashed curve of best fit is drawn to reveal the trend of variation. The inclusion of new data points for the PVME/ PCL and PVME/PHA blends (both polyesters with $\mathrm{CH}_{2} / \mathrm{CO}=5.0$ ) more precisely describes the trend of variation, and this confirms that the maximum interaction parameter remains the same at $\mathrm{CH}_{2} /$ $\mathrm{CO}=4.5$, as discussed in an earlier study. ${ }^{5}$ As the average polarity in polyesters decreases $\left(\mathrm{CH}_{2} / \mathrm{CO}=5.0\right.$ or higher $)$ or increases $\left(\mathrm{CH}_{2} /\right.$ $\mathrm{CO}=4.0$ or lower) from that of $\mathrm{PEAz}\left(\mathrm{CH}_{2} / \mathrm{CO}=4.5\right)$, the plot shows that the interaction parameter of the PVME/polyester decreases dramatically.

\section{Crystalline morphology and growth kinetics}

It has been reported that the blending of PCL with miscible amorphous polymers significantly changes the spherulite pattern. ${ }^{17}$ The effect of different diluent polymers varies. The inclusion of different amorphous polymers in PCL leads to different extents of suppression in growth rates and induces different spherulitic patterns. ${ }^{17}$ As an analogous polyester to PCL, PHA was used for comparison of spherulite growth in thin-film forms and the transformation of spherulite patterns in different regimes, both of which were investigated and analyzed. The spherulitic radius was found to increase linearly in time up to the point of spherulite impingement. The rejected, noncrystallizable PVME is most likely trapped between the growing fibrils of spherulites. Thus, the growth of the crystalline phase in neat PCL or PHA and its blends with PVME were examined to correlate with the behavior of the amorphous phase. Spherulite growth rates ( $G$ in $\mu \mathrm{m} \mathrm{min}^{-1}$ measured along a radial direction) as functions of $T_{\mathrm{c}}$ for neat PCL and PVME/PCL $=20 / 80$ were compared at several crystallization temperatures. Neat PCL had a greater growth rate than the $\mathrm{PVME} / \mathrm{PCL}=20 / 80$ blend at the same $T_{\mathcal{C}}$, indicating suppression of the growth rate by the diluent (PVME). For brevity, a figure of the growth data is not shown here. Growth data were further analyzed using the standard procedures of regime growth models. Regime analysis according to the Lauritzen-Hoffman (L-H) model ${ }^{18}$ for the growth rate of a crystallizing polymer is described as:

$$
G=G_{0} \exp \left[\frac{-U^{*}}{R\left(T_{\mathrm{c}}-T_{\infty}\right)}\right] \exp \left[\frac{-K_{\mathrm{g}}}{T_{\mathrm{c}} \Delta T_{f}}\right],
$$

where $U^{*}$ is the activation energy for the transportation of crystallizable segments to the crystallization front; $T_{\infty}$ is the temperature below which such molecular chain segmental motion ceases $\left(T_{\infty}=T_{\mathrm{g}}-30\right) ; \Delta T$ is the degree of undercooling $\left(\Delta T=T_{\mathrm{m}}^{\mathrm{o}}-T_{\mathrm{c}}\right) ; f$ is a correlation factor that accounts for the variation of the heat of fusion on temperature $\left(f=2 T_{\mathrm{c}} /\left(T_{\mathrm{m}}^{\mathrm{o}}+T_{\mathrm{c}}\right)\right)$; and $K_{\mathrm{g}}$ is the activation energy of nucleation for a crystal. The nucleation constant $K_{\mathrm{g}}$ can be expressed as

$$
K_{\mathrm{g}}=\frac{n b_{0} \sigma \sigma_{\mathrm{e}} T_{\mathrm{m}}^{\mathrm{o}}}{\Delta H^{\mathrm{o}} k}
$$

where $n$ is a constant equal to 4 for regime-I and -III and 2 for regimeII; $b_{0}$ is the thickness of single lamella (that is, a monolayer of lamella) in the direction normal to the growth plane; $\sigma$ and $\sigma_{\mathrm{e}}$ are the lateralsurface free energy and fold-surface free energy, respectively; and $k$ is the Boltzmann constant. It is convenient to rewrite Equation (3) to a logarithmic form for plotting, as

$$
\ln G+\left[\frac{U^{*}}{R\left(T_{\mathrm{c}}-T_{\infty}\right)}\right]=\ln G_{0}+\left[\frac{-K_{\mathrm{g}}}{T_{\mathrm{c}} \Delta T_{f}}\right],
$$

where $K_{\mathrm{g}}$ can be obtained by plotting the left-hand-side of Equation (5) vs $1 /\left(T_{\mathrm{c}} \Delta T f\right)$. 

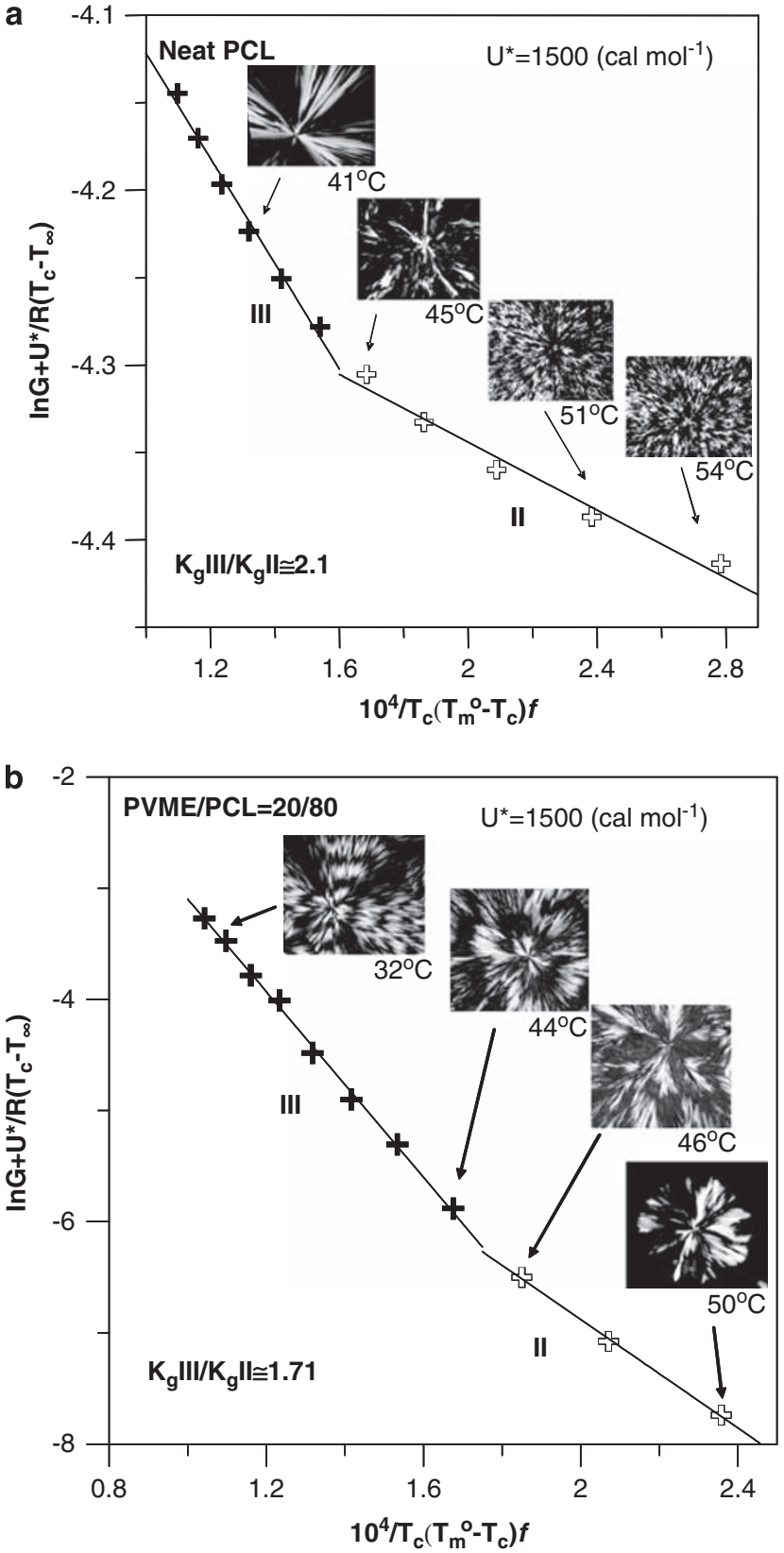

Figure 7 Regime-II to -III crystallization plots for (a) neat PCL and (b) PVME/PCL $=20 / 80$ blend by using $U^{*}=1500 \mathrm{cal} \mathrm{mol}^{-1}$. Insets: spherulite patterns for different regimes. A full color version of this figure is available at the Polymer Journal online.

Figures $7 \mathrm{a}$ and $\mathrm{b}$ shows Lauritzen-Hoffman plots for neat PCL in comparison with the PVME/PCL (20/80) blend, with insets showing POM graphs at specified temperatures. Figure $7 \mathrm{a}$ shows that PCL displays a transition from regime-II to -III. Inset graphs show the different Maltese-cross, dendrite or ring-band patterns in spherulites melt-crystallized at temperatures of different regimes. The slope change in this plot indicates that a transition from regime-II to -III (and not regime-I to -II) takes place in the temperature range covered. From the plot, a transition temperature is seen to be located at about $47^{\circ} \mathrm{C}$ (marking a division between regime-II and -III), where the pattern transforms from Maltese-cross spherulites (for $T_{\mathrm{c}}$ lower than $47^{\circ} \mathrm{C}$ ) to dendrites (feather like) with irregular zigzag rings (for $T_{\mathrm{c}}$ higher than $\left.47^{\circ} \mathrm{C}\right)$. When crystallized at lower $T_{\mathrm{c}}$ values, the spherulites in PCL are mostly ringless. Regime-III spherulites are mostly Maltese-cross patterned with no rings. Only at high temperatures of $T_{\mathrm{c}}=52-54{ }^{\circ} \mathrm{C}$ within regime-II does PCL crystallize into dendrite-like spherulites with rough and barely recognizable ring bands. Note that the ring band pattern in neat PCL is not distinct and seems to be quite irregular even at very high $T_{\mathrm{c}}$. Dendritic and ring characteristics increase with $T_{\mathrm{c}}$ within regime-II.

The spherulitic morphology and kinetic regime behavior for neat PCL were compared with those in the PVME/PCL blend of a representative composition $(w / w=20 / 80)$. Figure $7 b$ shows the Lauritzen-Hoffman plots displaying a transition from regime-II and -III for the PVME/PCL (20/80) blend. Note that striking differences in spherulite patterns are seen between the neat PCL (shown in the previous figure) and the PVME/PCL blend system (this figure). The blending of PCL with a miscible amorphous polymer (PVME) apparently results in significant changes in spherulite patterns. By contrast with neat PCL, the PVME/PCL blend at the miscible state is featured with spherulites of more regular and distinct rings. Again, the slope change in this plot for the PVME/PCL, similar to that for neat PCL, indicates that a transition from regime-II to -III takes place in the temperature range experimentally covered. For the PVME/PCL (20/80) blend, ring bands in spherulites are easily seen in crystallization at $T_{\mathrm{c}}=30-44^{\circ} \mathrm{C}$ (regime-III), but ring bands disappear on crystallization at $T_{\mathrm{c}}=46-50{ }^{\circ} \mathrm{C}$ (regime-II). Higher crystallization at $T_{\mathrm{c}}$ values $>50^{\circ} \mathrm{C}$ led to growth kinetics that were too slow for the blend morphology to be conveniently monitored; thus, this could not be performed. The regime kinetics transition is quite obvious, as the slope change is distinct between the two regimes, and an intersecting point is located at $T=46^{\circ} \mathrm{C}$. Thus, a trend is apparent for the PVME/ PCL $(20 / 80)$ blend, as discussed. Regime-III (below $T_{\mathrm{c}}=46^{\circ} \mathrm{C}$ ) is characterized with ring-band spherulites, whereas regime-II (above $46^{\circ} \mathrm{C}$ ) is featured with ringless spherulites. With further increases in $T_{\mathrm{c}}$ in regime-II, the pattern of spherulites continues to be ringless but becomes increasingly dendritic. Note that this behavior in the blend is opposite to the trend observed for neat PCL, in which regime-III is characterized with ringless spherulites and regime-II with dendritic/ irregular ring-banded spherulites, as discussed in the previous figure. The ring bands in the PVME/PCL blend for regime-III (low $T_{\mathrm{c}}$ ) are mostly zigzag with low regularity, which is similar to the pattern of ring bands seen in neat PCL crystallized at high $T_{\mathrm{c}}\left(52^{\circ} \mathrm{C}\right.$ or higher in regime-II). The amorphous and higher $T_{\mathrm{g}}$ PVME in the PVME/PCL blend (at $20 \mathrm{wt} \%$ loading in blend) 'retards' the original regime to a lower regime of growth.

The regime behaviors of neat PHA and miscible PVME/PHA blends were similarly analyzed for comparison with those for neat PCL or PVME/PCL blends. Figures $8 \mathrm{a}$ and $\mathrm{b}$ shows the Lauritzen-Hoffman plot for neat PHA in comparison with a PVME/PHA blend (20/80). The insets show the different Maltese-cross, dendrite or ring-band patterns in spherulites that were melt-crystallized at temperatures in different regimes. Interestingly, neat PHA and the PVME/PHA blend (20/80) display a similar transition from regime-I to -II, which is dramatically different from the regime-II to -III transition seen in neat PCL or PCL/PVME blends, as discussed earlier. The slope change suggests that neat PHA, in contrast to neat PCL, exhibits a transition from regime-I to -II (rather than from regime-II to -III). Crystal growth in regime-III, theoretically located at even lower $T_{\mathrm{c}}$ than regime-II, could not be experimentally verified for neat PHA. Thus, only regimes-I and -II were observed in PHA; by contrast, for PCL, only regimes-II and -III were observed, whereas regime-I could not be established. When crystallized at higher $T_{\mathrm{c}}$ values, PHA spherulites are 

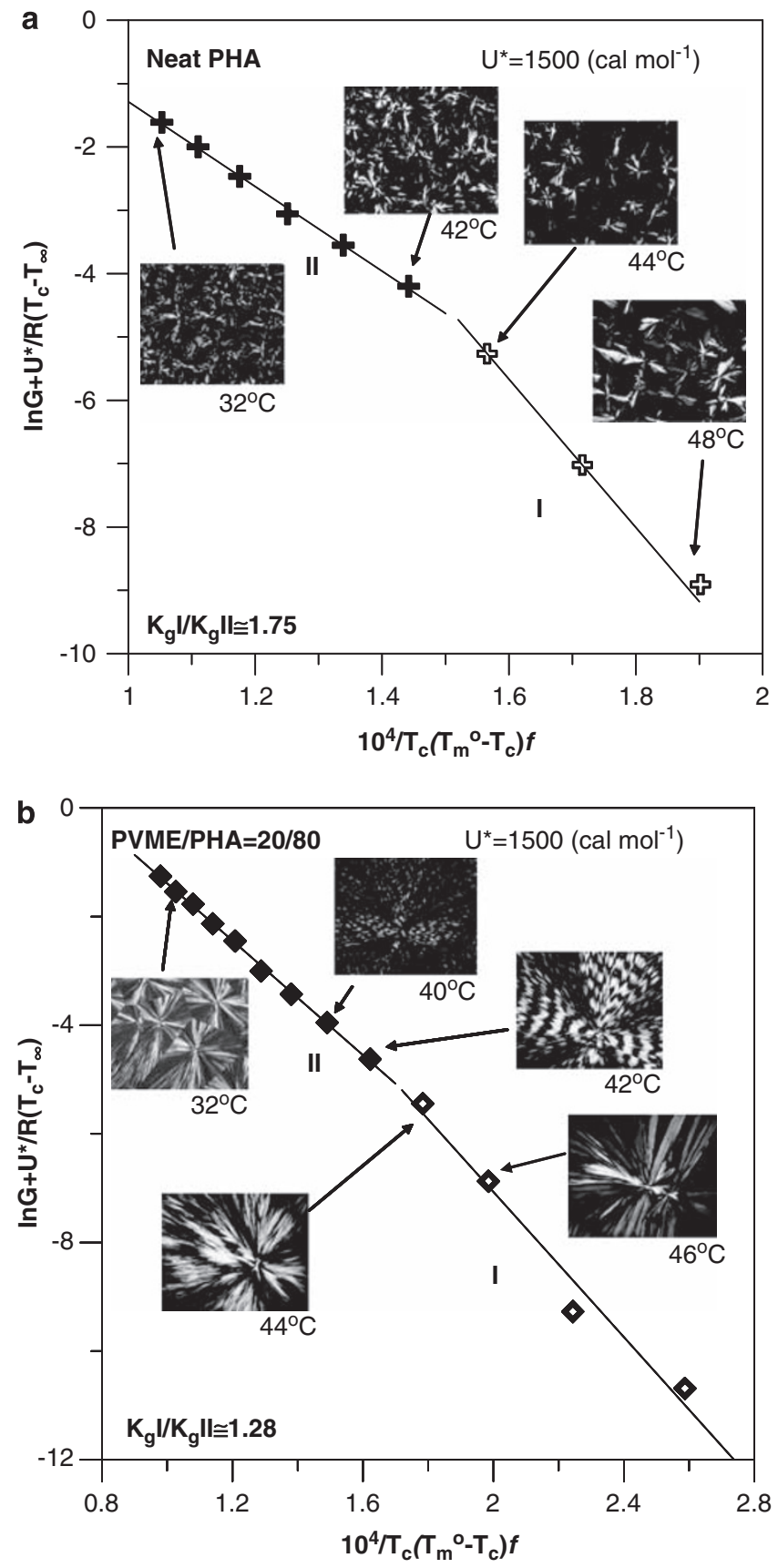

Figure 8 Regime-I to -II crystallizations plots for (a) neat PHA and (b) $\mathrm{PVME} / \mathrm{PHA}=20 / 80$ blend by using $U^{*}=1500 \mathrm{cal} \mathrm{mol}^{-1}$. Insets: spherulite patterns for different regimes. A full color version of this figure is available at the Polymer Journal online.

mostly ringless. Only at temperatures of $T_{\mathrm{c}}=32-42^{\circ} \mathrm{C}$ in regime-II did PHA crystallize into spherulites, showing rough and barely recognizable ring bands that were not very distinct. With further increases of $T_{c}$ to the range of regime-I, the ring-band feature in spherulites becomes increasingly less distinct and eventually disappears at higher $T_{\mathrm{c}}$. From the plot, a transition temperature is found at about $44^{\circ} \mathrm{C}$, which marks a division between regime-I and -II. Thus, a transformation trend in spherulite pattern is apparent for neat PHA. Regime-II (below $T_{\mathrm{c}}=44^{\circ} \mathrm{C}$ ) is characterized with ring-band spherulites, whereas regime-I (above $44^{\circ} \mathrm{C}$ ) features ringless spherulites.
A similar analysis was performed on the spherulitic morphology and kinetic regime behavior of the PVME/PHA blend of a representative composition $(\mathrm{w} / \mathrm{w}=20 / 80)$. Figure $8 \mathrm{~b}$ shows the LauritzenHoffman plot for the PVME/PHA (20/80) blend, the regime behavior of which is similar to neat PHA in that only regime-I and -II are seen. The regime kinetics transition is obvious, with an intersecting point located at $T=44^{\circ} \mathrm{C}$. Again, the way the slope changes in this plot suggests that the PVME/PHA blend, similar to neat PHA but in contrast to the neat PCL or PVME/PCL blend, exhibits a transition from regime-I to -II (rather than from regime-II to -III). The inset graphs in this figure show different patterns in spherulites that were melt-crystallized at the temperatures of the different regimes. Note that a striking difference in spherulite patterns is seen between the neat PHA and the PVME/PHA blend. For the PVME/PHA (20/80) blend, ring bands in spherulites are apparent in the crystallized blend films in a narrow temperature range of intermediate $T_{\mathrm{c}}=40-42{ }^{\circ} \mathrm{C}$ (regime-II), but the ring bands disappear in samples when crystallized at a low $T_{\mathrm{c}}=30-38{ }^{\circ} \mathrm{C}$ of regime-II or a high $T_{\mathrm{c}}=46-50{ }^{\circ} \mathrm{C}$ of regime-I; that is, not all $T_{\mathrm{c}}$ values in regime-II led to ring-banded spherulites, only $T_{\mathrm{c}}=40-42^{\circ} \mathrm{C}$ in regime-II did. A crystallization $T_{\mathrm{c}}>50{ }^{\circ} \mathrm{C}$ led to growth kinetics for the blend morphology that were too slow to be conveniently monitored, and thus such data were not available. Regime-I (above $44{ }^{\circ} \mathrm{C}$ ) featured mainly ringless spherulites. In regime-II, the blend featured either ring-banded spherulites or ringless ones (low $T_{\mathrm{c}}$ ). The ring-banded spherulites in the PVME/PHA blend that was crystallized at temperatures of $T_{\mathrm{c}}=40-42{ }^{\circ} \mathrm{C}$ exhibited much more distinct and regular ring bands than the spherulites seen in neat PHA.

\section{PVME/PHA vs PVME/PCL crystal morphology}

The amorphous and miscible PVME in the PVME/PHA blend apparently has a critical role in leading to differences in the major features of spherulites. Again, the effect of PVME in the PVME/PHA blend (at $20 \mathrm{wt} \%$ loading in the blend) seems to 'retard' the original regime to the next lower regime of growth, which is similar to the trend discussed for the PVME/PCL blend. The inclusion of amorphous PVME chains in the crystallizing species of PHA (in PVME/ PHA blend) or PCL (in PVME/PCL blend) tends to exert similar regime displacements. The changes induced by $20 \mathrm{wt} \%$ PVME in PVME/PHA or PVME/PCL blends are mostly twofold: (1) the elevation of the blend's $T_{\mathrm{g}}$, and (2) the depression of the $T_{\mathrm{m}}$ of the PHA or PCL in blended mixtures. Both factors may be responsible for the observed regime displacement and may contribute to changes in spherulite patterns. The miscibility behavior and interaction parameters in the amorphous phases are similar in these two PVME/PCL and PVME/PHA systems. However, the crystalline phases in these two blend systems had to be analyzed as well. Although the miscibilities in the amorphous phases of the PVME/PHA and PVME/PCL blends are quite similar, due to the isomeric nature of PHA and PCL, the crystalline morphologies are quite different between these two blends.

Figures 9a and b shows POM micrographs for the PVME/PHA (20/80) blend crystallized to four distinctly different morphologies at increasing $T_{\mathrm{c}}$ : Maltese-cross-type spherulites are seen at $T_{\mathrm{c}}=38^{\circ} \mathrm{C}$ (Figure 9a); ring-banded spherulites are obvious in the blend when crystallized at $T_{\mathrm{c}}=42{ }^{\circ} \mathrm{C}$ (Figure 9b); a strikingly different morphology of straight-stalk dendrites, resembling large chrysanthemum flower pestles, is present when the blend is crystallized at $T_{\mathrm{c}}=46^{\circ} \mathrm{C}$, and ultimately at $T_{\mathrm{c}}=46^{\circ} \mathrm{C}$ or above, dendrites remain straight but evolve into many more branched stalks, resembling banana stems. By comparison, neat PHA upon crystallization at all $T_{\mathrm{c}}$ values shows only Maltese-cross-type spherulites, differing from the PVME/PHA 

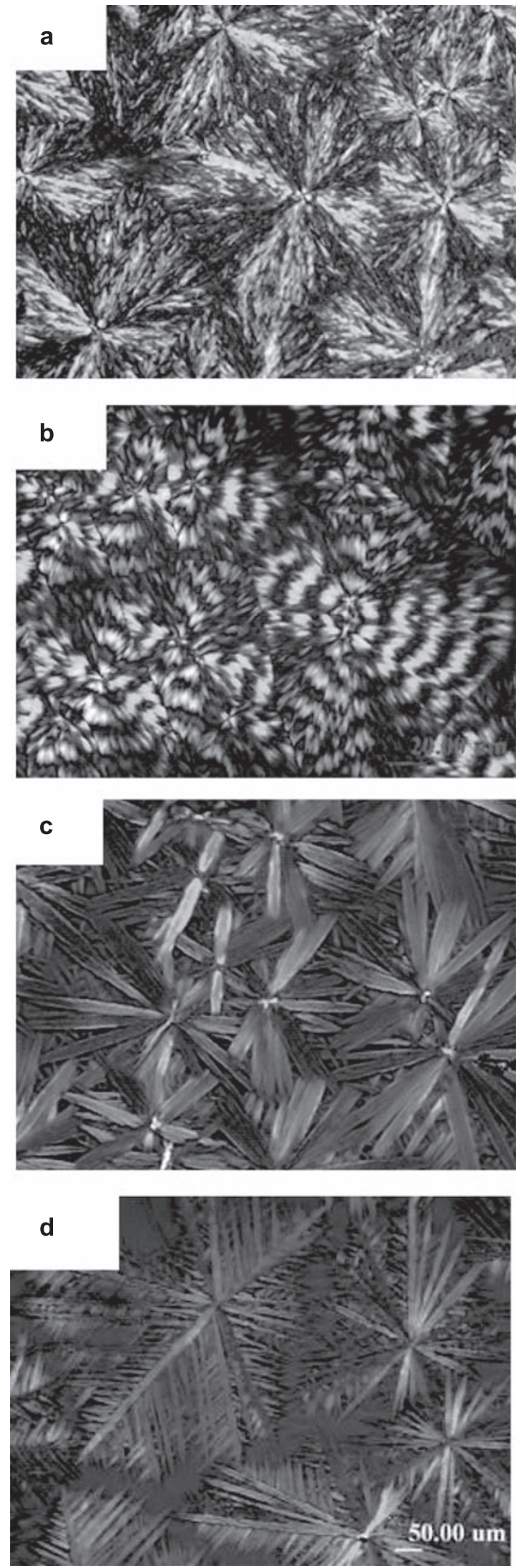

Figure 9 POM micrographs for the PVME/PHA (20/80) blend showing the transition of spherulite morphologies: (a) Maltese-cross type at $T_{\mathrm{c}}=38^{\circ} \mathrm{C}$ or lower, (b) ring-banded spherulites at $T_{\mathrm{c}}=42^{\circ} \mathrm{C}$, (c) straight-stalk dendrites at $T_{\mathrm{c}}=46^{\circ} \mathrm{C}$ and (d) branching dendrites at $T_{\mathrm{c}}=48^{\circ} \mathrm{C}$ or above. A full color version of this figure is available at the Polymer Journal online.
(20/80) blend only in the coarseness of the lamellae inside the spherulites. The PHA dendrites in the PVME/PHA blend are uniquely different in shape from the feather-like or snow-crystal dendrites reported in other polymers or blends. The PHA dendrites in the PVME/PHA (20/80) blend resemble chrysanthemum flower pestles, showing almost no branches from the radial, straight stalks, and they assume the main characteristics of single crystals. Some natural rocks solidified slowly from molten lava are also known to pack into such single crystals, assuming large chrysanthemum flower pestles. Note also that the color contrast divides dendritic straight stalks into four quadrants, with roughly three to four stalks in each quadrant. The PVME in the PVME/PHA (20/80) blend is responsible for the dramatic changes in morphology patterns, leading to the single-crystal straight stalks at high $T_{\mathrm{c}}$. This fact suggests that highly suppressed growth rates, retarded by miscible PVME and high $T_{\mathcal{c}}$, are the main factors influencing the feasibility of PHA polymer chains packing into large-dimension single crystals from bulk forms.

\section{CONCLUSION}

In this study, detailed comparisons were performed for the phase behavior and crystalline morphology (ring-banded spherulites and unusual dendritic lamellae) in blends of PVME with two structurally similar polyesters (PCL vs PHA, which are chemically isomeric with the same $\mathrm{CH}_{2} / \mathrm{CO}$ ratio=5) of different crystallizing tendencies and of different crystalline morphologies. The amorphous phases in both PVME/PCL and PVME/PHA blends were proven to be miscible, with weak and nonspecific interactions. Both blend systems exhibited similar $T_{\mathrm{g}}$-composition relationships, and the interaction strengths were similar $(\chi=-0.20$ vs -0.21$)$, indicating similar extents of phase homogeneity. However, the behavior and morphology of the crystal phases in these two blends differed. For the PVME/PHA blend, wherein the PHA's crystallinity is much harder to suppress on quenching than that of the PCL in blends of the same proportions, the extent of positive $T_{\mathrm{g}}$ deviation from linearity was proportionally more significant and the composition range of such deviation was wider than for the PVME/PCL blend. The $T_{\mathrm{g}}$ behavior in blends served to validate the effects of residual crystallinity (remaining after quenching) on observing the abrupt positive deviation noted in the $T_{\mathrm{g}}$-composition relationships for several PVME/polyester blends.

Neat PHA shows a regime-I to -II transition, during which its spherulite pattern remains similarly Maltese-cross type. On blending with PVME (at $20 \mathrm{wt} \%$ ), the PVME/PHA blend undergoes dramatic changes. For the low $T_{\mathrm{C}}$ range $\left(32-42{ }^{\circ} \mathrm{C}\right.$ within regime-II), the PVME/ PHA blend assumes either original Maltese-cross-type or ringbanded-type spherulites, but it transforms into highly dendritic lamellae for the high $T_{\mathrm{c}}$ range $\left(46-48^{\circ} \mathrm{C}\right.$ within regime-I). Dendrites in the high- $T_{\mathrm{c}}$-crystallized PVME/PHA blend assume a straight-stalk pattern (chrysanthemum flower pestles), which is not seen in neat PHA. The unusual morphology in the PHA blend indicates a significant effect of PVME on PHA morphology. By comparison, neat PCL shows a regime-II to -III transition, wherein its spherulite pattern transforms from a Maltese-cross type for a low range of $T_{\mathrm{c}}$ to a fluffy dendritic type for extremely high $T_{\mathrm{c}}$ values approaching $T_{\mathrm{m}}$. On blending with PVME (at $20 \mathrm{wt} \%$ ), the PVME/PCL blend undergoes changes. For the low $T_{\mathrm{c}}$ range $\left(28 \sim 40{ }^{\circ} \mathrm{C}\right.$, in regime-III), the PVME/ PCL blend assumes ring-banded-type spherulites, but it transforms to a fluffy dendritic lamellae pattern for the high $T_{\mathrm{c}}$ range $\left(45-50^{\circ} \mathrm{C}\right.$, in regime-II). The morphologies in the crystalline phases of these two blend systems differ significantly. Dendrites for the high- $T_{\mathrm{c}}\left(46^{\circ} \mathrm{C}\right.$ or above)-crystallized PVME/PCL blend assume a fluffy feather pattern, which is dramatically different from the straight-stalk dendrites in the 
PVME/PHA blend. However, the crystalline behaviors of both miscible blend systems exhibit similarity in that they go through a regime transition at a certain temperature $\left(45^{\circ} \mathrm{C}\right)$, a break point above or below which the spherulite patterns transform from Maltese-cross to dendritic types.

\section{ACKNOWLEDGEMENTS}

This work was financially supported by a basic research grant (NSC-95 2221 E006 183 and NSC 96-2221-E-006-099-MY3) in consecutive years from National Science Council (NSC) of Taiwan.

1 Yam, W. Y., Ismail, J., Kammer, H. W., Schmidt, H. \& Kummerlöwe, C. Polymer blends of poly( $\varepsilon$-caprolactone) and poly(vinyl methyl ether) - thermal properties and morphology. Polymer 40, 5545-5552 (1999).

2 Guo, Q. Completely miscible ternary blends poly(hydroxyether of bisphenal-A)-poly(vinyl methyl ether)-poly(e-caprolactone). Eur. Polym. J. 26, 1329-1332 (1990)

3 Oudhuis, A.A.C.M., Thiewes, H. J., van Hutten, P. F. \& ten Brinke, G A comparison between the morphology of semicrystalline polymer blends of poly( $\varepsilon$-caprolactone)/ poly(vinyl methyl ether) and poly( $\varepsilon$-caprolactone)/(styrene-acrylonitrile). Polymer 35, 3936-3942 (1994).

4 Bisso, G., Casarino, P. \& Pedemonte, E. Thermodynamics of polymer blends based on poly(E-caprolactone)/poly(vinyl methyl ether). Macromol. Chem. Phys. 200, 376-383 (1999).

5 Chiang, W. J. \& Woo, E. M. Comparison of glass transition and interpretation on miscibility in blends of amorphous poly(vinyl methyl ether) with highly crystallizable versus less-crystallizable polyesters. J. Polym. Sci., Polym. Phys. B 45, 2899-2911 (2007)
6 Brode, G. L. \& Koleske, J. V. Lactone polymerization and polymer properties. J. Macromol. Sci. Part A. 6, 1109-1144 (1972).

7 Wang, J., Cheung, M. K. \& Mi, Y. Miscibility and morphology in crystalline/amorphous blends of poly(caprolactone)/poly(4-vinylphenol) as studied by DSC, FTIR, and ${ }^{13} \mathrm{C}$ solid state NMR. Polymer 43, 1357-1364 (2002).

8 Yen, K. C., Mandal, T. K. \& Woo, E. M. Enhancement of bio-compatibility via specific interactions in polyesters modified with a bio-resourceful macromolecular ester containing polyphenol groups. J. Biomed. Mater. Res. Part A 86, 701-712 (2008).

9 Robeson, L. M., Hale, W. F. \& Merriam, C. N. Miscibility of the poly(hydroxy ether) of bisphenol A with water-soluble polyethers. Macromolecules 14, 1644-1650 (1981).

10 Pedrosa, P., Pomposo, J. A., Calahorra, E. \& Cortazar, M. On the glass transition behavior, interaction energies, and hydrogen-bonding strengths of binary poly( $p$-vinylphenol)/polyether blends. Macromolecules 27, 102-109 (1994).

11 Chakraborty, S. S., Maiti, N., Mandal, B. M. \& Bhattacharyya, S. N. Miscibility and phase diagrams for poly(vinyl methyl ether) and polyacrylate blend systems. Polymer 34, 111-114 (1993).

12 Mandal, T. K. \& Woo, E. M. Marginal miscibility and solvent-dependent phase behavior in solution-blended poly(vinyl methyl ether)/poly(benzyl methacrylate). Macromol. Chem. Phys. 200, 1143-1149 (1999).

13 Flory, P. J. in Principles of Polymer Chemistry (ed. Flory, P.J.) Ch. XII (Cornell University Press, Ithaca, NY, USA, 1978).

14 Nishi, T. \& Wang, T. T. Melting point depression and kinetic point effects of cooling on crystallization in poly(vinylidene fluoride)-poly(methyl methacrylate) mixtures. Macromolecules 8, 909-915 (1975).

15 Ahmad, H. \& Yaseen, M. in Polymer Handbook (eds Brand, J., Immergut, E.H., Grulke, E.A.) Chap.VII, 706, (Wiley, New York, USA, 1999).

16 Woo, E. M., Mandal, T. K. \& Lee, S. C. Relationships between ringed spherulitic morphology and miscibility in blends of poly( $\varepsilon$-caprolactone) with poly(benzyl methacrylate) versus poly(phenyl methacrylate). Colloid. Polym. Sci. 278, 1032-1042 (2000).

17 Chen, Y. F. \& Woo, E. M. Growth regimes and spherulites in thin-film poly( $\varepsilon$-caprolactone) with amorphous polymers. Colloid. Polym. Sci. 286, 917-926 (2008).

18 Hoffman, J. D. Regime III crystallization in melt-crystallized polymers: the variable cluster model of chain folding. Polymer 24, 3-26 (1983). 